\title{
Preferencia de Tadalafilo vs Sildenafilo en pacientes españoles con disfunción eréctil: resultados procedentes de un estudio multicéntrico internacional
}

\author{
L. Rodríguez Vela*, E. Lledó García**, O. Rajmil***, D. Mo***, A. Cassinello*****, \\ J. Casariego*****
}

*Hospital Miguel Servet, Zaragoza. **Hospital Gregorio Marañón, Madrid. ***Fundación Puigvert, Barcelona. ${ }^{* * * *}$ Eli Lilly \& Co. Indianapolis (Indiana). EEUU. ${ }^{* * * * * L i l l y ~ S . A . ~ A l c o b e n d a s ~(M a d r i d) ~}$

Actas Urol Esp 2006; 30 (1): 67-79

\section{RESUMEN}

PREFERENCIA DE TADALAFILO VS SILDENAFILO EN PACIENTES ESPAÑOLES CON DISFUNCIÓN ERÉCTIL: RESULTADOS PROCEDENTES DE UN ESTUDIO MULTICÉNTRICO INTERNACIONAL

Fundamento y objetivo: Comparar la preferencia por sildenafilo vs. tadalafilo, y por sus instrucciones de administración en una cohorte de pacientes españoles con disfunción eréctil (DE).

Material y método: 64 pacientes españoles participantes en un estudio multicéntrico, de 2 periodos, cruzado y doble-ciego (con 265 pacientes en total) se distribuyeron aleatoriamente para recibir sildenafilo (50 mg) o tadalafilo $(20 \mathrm{mg}$ ) a demanda durante 12 semanas, tras lo cual se cruzaron para recibir el régimen alternativo otras 12 semanas, para valorar la preferencia por uno de los dos tratamientos en una fase de extensión del estudio. Igualmente, para valorar la preferencia por las respectivas instrucciones de administración, 13 pacientes se distribuyeron en 2 brazos de tratamiento con tadalafilo 20 mg: uno con las instrucciones de administración de sildenafilo (S) y otro con las de tadalafilo (T).

Resultados: De los 56 pacientes que finalizaron el estudio, un $70 \%$ eligieron recibir tratamiento con tadalafilo frente a sildenafilo (30\%) en la extensión ( $\mathrm{p}<0,01)$. Igualmente, un $73 \%$ de los 13 que valoraron las instrucciones de administración de ambos fármacos prefirieron las instrucciones de $\mathrm{T}$ ( $\mathrm{p}>0,05)$. La preferencia no varió con la edad, enfermedades concomitantes o uso previo de sildenafilo.

Conclusiones: En este estudio, aproximadamente 7 de cada 10 pacientes prefirieron tadalafilo y sus instrucciones de administración frente a sildenafilo, como tratamiento para su DE.

Palabras clave: Disfunción eréctil. Tadalafilo. Sildenafilo. Inhibidores de fosfodiesterasa. Estudio de preferencia. Ensayo clínico aleatorizado.

\section{ABSTRACT \\ TADALAFIL VS SILDENAFIL PATIENT PREFERENCE IN SPANISH MEN WITH ERECTILE DYSFUNCTION: RESULTS FROM AN INTERNATIONAL MULTICENTRIC STUDY}

Objective: To compare patient preference for sildenafil citrate (sildenafil) vs. tadalafil and for their respective dosing instructions in a cohort of Spanish patients with erectile dysfunction (ED).

Material and methods: Sixty four Spanish patients from a multicenter, two period, cross-over, double-blind study (265 patients enrolled in total) were randomized to receive on-demand sildenafil $50 \mathrm{mg}$ or tadalafil $20 \mathrm{mg}$ for 12 weeks and afterwards were crossed over to the alternate regimen for another 12 weeks to assess drug preference in an extension period of the study. Similarly, to evaluate preference for their respective dosing instructions, 30 patients were randomized to one of the 2 arms treated with tadalafil: one with sildenafil (S) dosing instructions and the other with tadalafil (T) dosing instructions.

Results: Seventy percent of 56 patients completing the study chose to receive tadalafil treatment versus sildenafil treatment $(30 \%)$ in the extension period $(\mathrm{p}<0.01)$. Correspondingly, $73 \%$ of 13 evaluating each drug dosing instructions preferred $\mathrm{T}$ dosing instructions ( $\mathrm{p}>0.05$ ). Preference did not vary with age, concomitant diseases and previous use of sildenafil.

Conclusions: In this study, 7 out of 10 patients preferred tadalafil and its dosing instructions to sildenafil, for the treatment of their ED.

Keywords: Erectile dysfunction. Tadalafil. Sildenafil. Phosphodiesterase inhibitors. Preference study. Randomized clinical trial. 
L a disfunción eréctil (DE), se define como la incapacidad para conseguir o mantener una erección con suficiente rigidez para permitir una relación sexual satisfactoria, afecta a un importante sector de la población masculina y conduce, entre otros, a un deterioro psicológico de la propia imagen, de las relaciones de pareja y de la calidad de vida ${ }^{1-3}$. Según la OMS, la prevalencia estimada de DE para los años 2004, 2005, y 2006 se aproxima a los 189, 193, y 198 millones de varones afectados en todo el mundo ${ }^{4}$ y unos 2 millones en España ${ }^{5}$. Con el envejecimiento progresivo de la población mundial, se estima que esta alta prevalencia pueda llegar a duplicarse en $2025^{6}$. Actualmente, los inhibidores de la fosfodiesterasa 5 (PDE5) son considerados como el primer escalón en el tratamiento de la DE para la mayoría de los pacientes, por su alta eficacia, buena tolerabilidad y facilidad de administración ${ }^{7}$. Si bien comparten mecanismo de acción y un perfil de eficacia y seguridad semejante, presentan entre sí diferencias clínicas relevantes, en cuanto a la duración del periodo de respuesta e interacción con alimentos se refiere, fundamentalmente.

El citrato de sildenafilo (Viagra, Pfizer), comercializado desde 1998, es el más antiguo de estos fármacos orales, y ha demostrado eficacia en los ensayos clínicos de disfunción eréctil y también en la práctica clínica ${ }^{8}$ y constituye el máximo exponente de los inhibidores de PDE5 de acción corta, ya que su eficacia tiene una duración aproximada de 4-5 horas. La ingesta de alimentos puede afectar la magnitud de su absorción y retrasar su inicio de acción ${ }^{9}$. A pesar de presentar altos niveles de eficacia y ser, en general, bien tolerado a las dosis habituales, se ha observado que entre un 20-50\% de los pacientes que responden a sildenafilo abandonan a largo plazo el tratamiento por diversos motivos ${ }^{10}$, hecho que alerta sobre la existencia y persistencia de necesidades no cubiertas por el tratamiento en pacientes con DE. Tadalafilo (Cialis, Lilly ICOS) es un inhibidor de la PDE5, aprobado como tratamiento oral de la DE en $2002^{11}$. En los estudios clínicos realizados ha demostrado ser un tratamiento eficaz y bien tolerado para el tratamiento de la disfunción eréctil ${ }^{12-14}$. Sus propiedades farmacocinéticas singulares (vida media de 17,5 horas) se traducen clínicamente en un periodo más largo de respuesta, de modo que algunos hombres han experimentado relaciones sexuales satisfactorias tan pronto como a los 16 minutos tras la toma y hasta 36 horas después ${ }^{1-13,15-17}$. A diferencia de sildenafilo, la absorción de tadalafilo no se ve afectada por la ingesta de alimen$\operatorname{tos}^{11}$.

La medicina del S XXI, impulsa la implicación activa del paciente en la toma de decisiones clínicas, frente al principio de autoridad en el que se basaba el ejercicio tradicional de la medicina. Especialmente en el caso de la DE, existen múltiples factores sociológicos, psicológicos, culturales y relacionados con los hábitos sexuales que deben tenerse en cuenta a la hora de indicar un determinado tratamiento, pues éste debería ajustarse en la medida de lo posible a los requerimientos, expectativas y necesidades del paciente y su pareja. Es decir, ajustarse a las preferencias de los pacientes. Así, en el momento actual y una vez demostrada la eficacia de estos fármacos en estudios diseñados convenientemente a tal efecto y empleando las escalas de medición de la eficacia tradicionalesÍndice Internacional de Función Eréctil (IIEF), diario del acto sexual (SEP) y pregunta Global de Eficacia (GAQ)-, se está concediendo gran importancia a la evaluación de las preferencias y necesidades de los pacientes para complementar la información sobre eficacia y seguridad comparativas $^{18}$. Se han realizado varios estudios que han evaluado tanto la preferencia de los pacientes por diferentes modalidades de tratamiento para la $\mathrm{DE}^{18,19}$, como algunos estudios de preferencia que comparan distintos inhibidores de PDE5, empleando diseños y metodología variados $^{20-24}$. La gran mayoría de estos estudios evidencian una clara preferencia de los pacientes por tadalafilo empleando un diseño abierto, es decir, sin enmascaramiento de la medicación a estudio, por la complejidad que suponía preservar el doble ciego en la comparación de dos fármacos con distintas instrucciones de administración. 
Se diseñó un estudio que evaluase las preferencias de los pacientes por tadalafilo o sildenafilo, así como la preferencia por las distintas instrucciones de administración, pero empleando una metodología especial de enmascaramiento que preservase el ciego $^{24}$. El presente trabajo recoge los resultados obtenidos en una cohorte de pacientes españoles con DE que participaron en este estudio de preferencia, realizado en EEUU y Europa y que incluyó una muestra total de 265 pacientes con DE. Los resultados globales de este estudio fueron publicados por Von Keitz et al. ${ }^{24}$, de manera que este artículo se centrará en la muestra española participante.

\section{MATERIAL Y MÉTODOS}

\section{Métodos}

A 84 pacientes con disfunción eréctil atendidos en diversos centros hospitalarios españoles se les ofreció la posibilidad de participar en un estudio multicéntrico, cruzado, de dos periodos, doble ciego y aleatorizado, que englobaba un total de 265 pacientes de 15 centros en Estados Unidos, España y Alemania. El estudio se realizó cumpliendo los principios éticos de la declaración de Helsinki, así como las leyes y regulaciones aplicables en cada país. El protocolo fue revisado y aprobado en cada centro por un comité de revisión ética independiente y por la Agencia Española del Medicamento. Todos los pacientes dieron su consentimiento informado por escrito.

\section{Pacientes}

Se consideró elegibles para la inclusión a los varones de 18 a 66 años de edad que mantenían relaciones sexuales con una pareja femenina, y que presentaban una DE de al menos 3 meses de evolución. La DE se definió como un cambio mantenido en la calidad de la erección que influía de modo negativo en la satisfacción del paciente respecto a la relación sexual. Los pacientes elegibles aceptaron no utilizar ninguna otra forma de tratamiento para DE durante todo el periodo de estudio, incluyendo los periodos de valoración inicial, tratamiento cruzado y extensión, y ni durante las 96 horas siguientes a la finalización del periodo de extensión. Se aceptó la participa- ción en el estudio de pacientes que no habían respondido previamente a sildenafilo. Se excluyó del estudio a los pacientes con eyaculación precoz, DE debida a un trastorno endocrinológico no tratado (por ejemplo, hipopituitarismo, hipotiroidismo o hipogonadismo), antecedentes de cirugía pélvica sin signos de preservación de la función eréctil, ictus o lesiones medulares en los 6 meses previos, antecedentes de infección por VIH, tratamiento actual con nitratos, infarto de miocardio en los 90 días previos, revascularización coronaria en los 90 días previos, o antecedentes de angina inestable en los 6 meses previos. Se excluyó también a los pacientes con retinitis pigmentaria, puesto que el prospecto de sildenafilo incluye una advertencia contraria a su uso.

\section{Diseño del estudio}

El objetivo principal del estudio fue la Valoración de la Preferencia por la Medicación (PM) y se evaluó en una cohorte de pacientes con DE que recibieron, alternativamente, sildenafilo a una dosis inicial de $50 \mathrm{mg}$, y tadalafilo $20 \mathrm{mg}$, durante 12 semanas, respectivamente, según un diseño cruzado. La Valoración de la Preferencia por las Instrucciones de Administración (PIA) se llevó a cabo en una cohorte distinta de pacientes, tratados con tadalafilo $20 \mathrm{mg}$ con el objeto de evaluar su preferencia por el empleo de tadalafilo bajo los condicionamientos impuestos por las instrucciones de administración propias de sildenafilo y las de tadalafilo. Tanto en la Valoración de Preferencia por la Medicación como en la Valoración de Preferencia por las Instrucciones de Administración, a todos los pacientes que seguían las instrucciones de administración de sildenafilo se les ofrecía la posibilidad de aumentar la dosis después de 4 semanas de tratamiento. Con un diseño doble ciego, todos los pacientes que solicitaron un ajuste de dosis al alza recibieron cápsulas adicionales. El número de pacientes que recibieron medicación activa adicional en diseño doble ciego se limitó al 35\% del total de pacientes tratados con sildenafilo en cada periodo de tratamiento en cada país. Los demás pacientes del grupo de sildenafilo y todos los del grupo de tadalafilo que solicitaron el ajuste al alza recibieron un placebo en diseño doble ciego. El límite del 
número de pacientes tratados con sildenafilo que podía aumentar la dosis se estableció para simular el patrón de uso observado en la práctica clínica (es decir, una estimación basada en los datos existentes sobre la proporción de pacientes que toman sildenafilo que aumentan realmente sus dosis) ${ }^{25-27}$.

$\mathrm{Al}$ concluir el Periodo de Tratamiento Cruzado de 12 semanas, se preguntó a los pacientes sobre cuál de los dos tratamientos recibidos preferían recibir durante un período de extensión opcional de 12 semanas, manteniendo el doble ciego y sin coste alguno para los pacientes, con objeto de eliminar el coste de adquisición como variable que pudiera determinar la preferencia. El Periodo de Extensión fue precedido de un periodo de lavado farmacológico de 96 horas.
Tanto durante el Periodo de Tratamiento Cruzado como durante el Periodo de Extensión (Fig. 1), los pacientes registraron la fecha y hora en que tomaban el fármaco en estudio utilizando un diario para la medicación, así como la fecha y hora de los intentos de relación sexual en un diario de relaciones sexuales.

\section{Ramas de tratamiento y diseño ciego}

El protocolo proporcionado a los investigadores establecía que, después de un periodo de valoración inicial sin tratamiento de aproximadamente una semana, debía asignarse aleatoriamente a los pacientes a los grupos de tratamiento con tadalafilo $20 \mathrm{mg}$ (utilizando las instrucciones de administración de sildenafilo o de tadala-

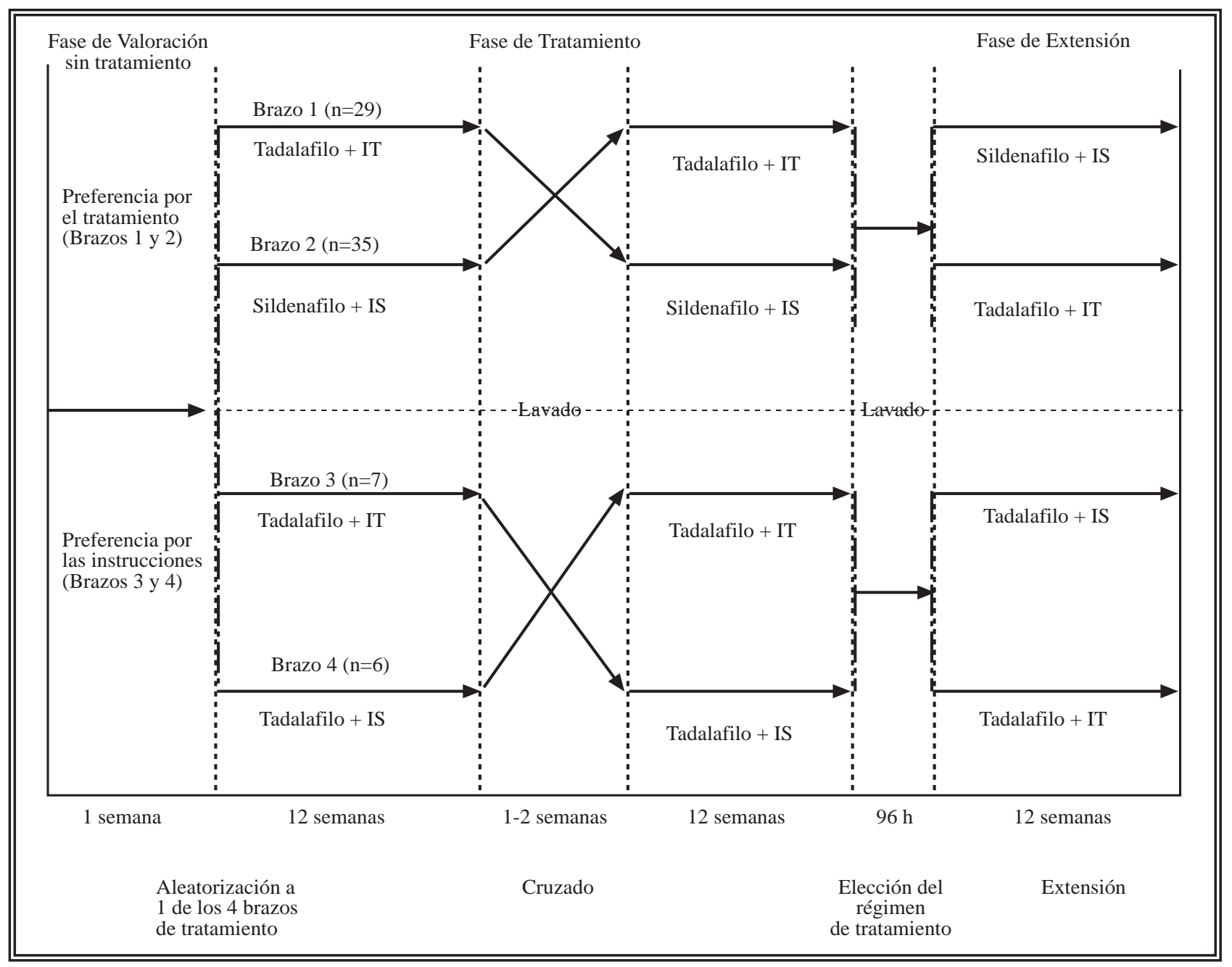

FIGURA 1. Diseño del estudio multicéntrico.

IT: Instrucciones de administración de Tadalafilo.

IS: Instrucciones de administración de Sildenafilo. 
filo), sildenafilo $50 \mathrm{mg}$ (con las instrucciones de administración de sildenafilo solamente) o placebo (con las instrucciones de administración de sildenafilo o de tadalafilo). El protocolo indicaba también que los pacientes debían recibir un tratamiento activo en al menos una de las fases del Periodo de Tratamiento Cruzado. En realidad, ninguno de los pacientes recibió placebo. Se asignó los pacientes aleatoriamente a las distintas ramas de tratamiento para la Valoración de la Preferencia por la Medicación o a la Valoración de Preferencia por las Instrucciones de Administración para el tratamiento con tadalafilo. La información relativa a la rama de tratamiento real se proporcionó exclusivamente a cada uno de los comités de revisión ética, como suplemento del protocolo.

Las ramas simuladas de placebo constituyen una metodología especial que permite enmascarar la asignación de tratamiento cuando se utilizan dos instrucciones de administración diferentes para dos fármacos con perfiles farmacocinéticos distintos. Los pacientes tratados con sildenafilo recibieron tan sólo las instrucciones de administración correspondientes a este fármaco, ya que administrarlo con las de tadalafilo probablemente influiría negativamente en la percepción de eficacia si el paciente desarrolla actividad sexual más allá de las 4 horas tras la dosis o si ingiere alimento. Por contra, los pacientes tratados con tadalafilo recibieron las instrucciones de administración de sildenafilo o de tadalafilo, pues el perfil clínico de tadalafilo es compatible con ambas. Resulta evidente que, sin las ramas simuladas de placebo, la entrega de las instrucciones de administración de tadalafilo hubiera eliminado el diseño ciego respecto a los pacientes y sus médicos, pues hubiese significado que el paciente estaba recibiendo, indefectiblemente, tadalafilo. Además, la medicación en estudio de sildenafilo y tadalafilo se administró en cápsulas idénticas para garantizar el diseño ciego. La encapsulación no influyó de manera significativa en la disolución de ambos fármacos.

\section{Instrucciones de administración}

Las instrucciones de administración de sildenafilo se basaron en las instrucciones proporcio- nadas por el fabricante ${ }^{9}$ y se entregaron para maximizar la eficacia de sildenafilo. Las instrucciones de administración de tadalafilo se basaron en las instrucciones utilizadas en ensayos clinicos previos con este fármaco y en el tipo de lenguaje desarrollado para los pacientes ${ }^{24}$. El prospecto de sildenafilo aconseja al paciente tomar la medicación una hora antes de la actividad sexual, pero indica que la medicación es efectiva entre 30 minutos y 4 horas tras su ingesta. En cambio, la mayor duración del efecto de tadalafilo se incluyó en las instrucciones de administración utilizadas en este estudio (véase el comentario más adelante).

\section{Variables de valoración}

La variable de valoración principal fue la preferencia de los pacientes respecto al tratamiento de la DE- tadalafilo o sildenafilo-, evaluado mediante la elección del paciente, con un diseño ciego, utilizados con sus respectivas instrucciones de administración, para la medicación del Periodo de Extensión. Las variables secundarias fueron la preferencia de los pacientes respecto a las instrucciones de administración de tadalafilo o sildenafilo durante el tratamiento con tadalafilo, y el tiempo transcurrido entre la toma del medicamento y el intento de relación sexual. Las valoraciones de la seguridad incluyeron una anamnesis completa, exploración física, electrocardiograma de 12 derivaciones y análisis de bioquímica sérica y hematología estándar en la visita de selección inicial. Se registró la presión arterial, el pulso y la incidencia de acontecimientos adversos en cada visita ambulatoria. Los acontecimientos adversos se resumieron según el Medical Dictionary for Regulatory Activities (MedDRA versión 4,0) para la intensidad y la relación con el fármaco en estudio.

\section{Análisis estadísticos}

En este artículo sólo se analizan los datos de los pacientes españoles que participaron en el estudio. El análisis de preferencia por la medicación incluye los pacientes que decidieron continuar recibiendo tratamiento para su $\mathrm{DE}$ en el periodo de extensión. Se empleó un test $z$ bilate- 
ral para probar la hipótesis nula de que la proporción de pacientes que escogían tadalafilo o sildenafilo al inicio del periodo de extensión era igual, con un nivel de significación estadística de 0,05 .

El cálculo del tamaño muestral estimaba que 56 pacientes que respondieran a la pregunta de preferencia permitía una potencia estadística del $87 \%$ par rechazar la hipótesis nula, considerando que cerca del $70 \%$ de los pacientes escogerían tadalafilo. Una muestra de 64 pacientes aleatorizados con una tasa de finalización del estudio del $88 \%$ se consideró suficiente para la valoración de la preferencia por la medicación. Se empleó un test chi- cuadrado para valorar la distribución de preferencia entre pacientes españoles y no españoles. Se realizó un análisis descriptivo para la distribución temporal de los intentos de coito. El número total de intentos de coito y porcentaje de intentos de coito a lo largo del tiempo (en horas) tras la toma de la medicación, se recogen según fármaco administrado.

$\mathrm{El}$ análisis de seguridad incluye un resumen de eventos adversos por tratamiento, para todos los pacientes incluidos en asignación aleatoria.

\section{RESULTADOS}

\section{CARACTERÍSTICAS DE LOS PACIENTES}

De los 84 pacientes españoles incluidos, 77 fueron aleatorizados de forma que 64 se distribuyeron al azar entre las dos ramas de valoración de preferencia por un tratamiento (PT) y 13 pacientes se distribuyeron entre las dos ramas de valoración por las instrucciones de administración (PIA) (Fig. 2). Completaron el periodo cruzado 56 de los 64 pacientes de las ramas 1 y 2 de tratamiento (87,5\%); y 11 de los $13(84,6 \%)$ de las ramas 3 y 4 , los cuales optaron por continuar en la fase de extensión con el tratamiento recibido en la fase doble-ciego. Las razones por las cuales

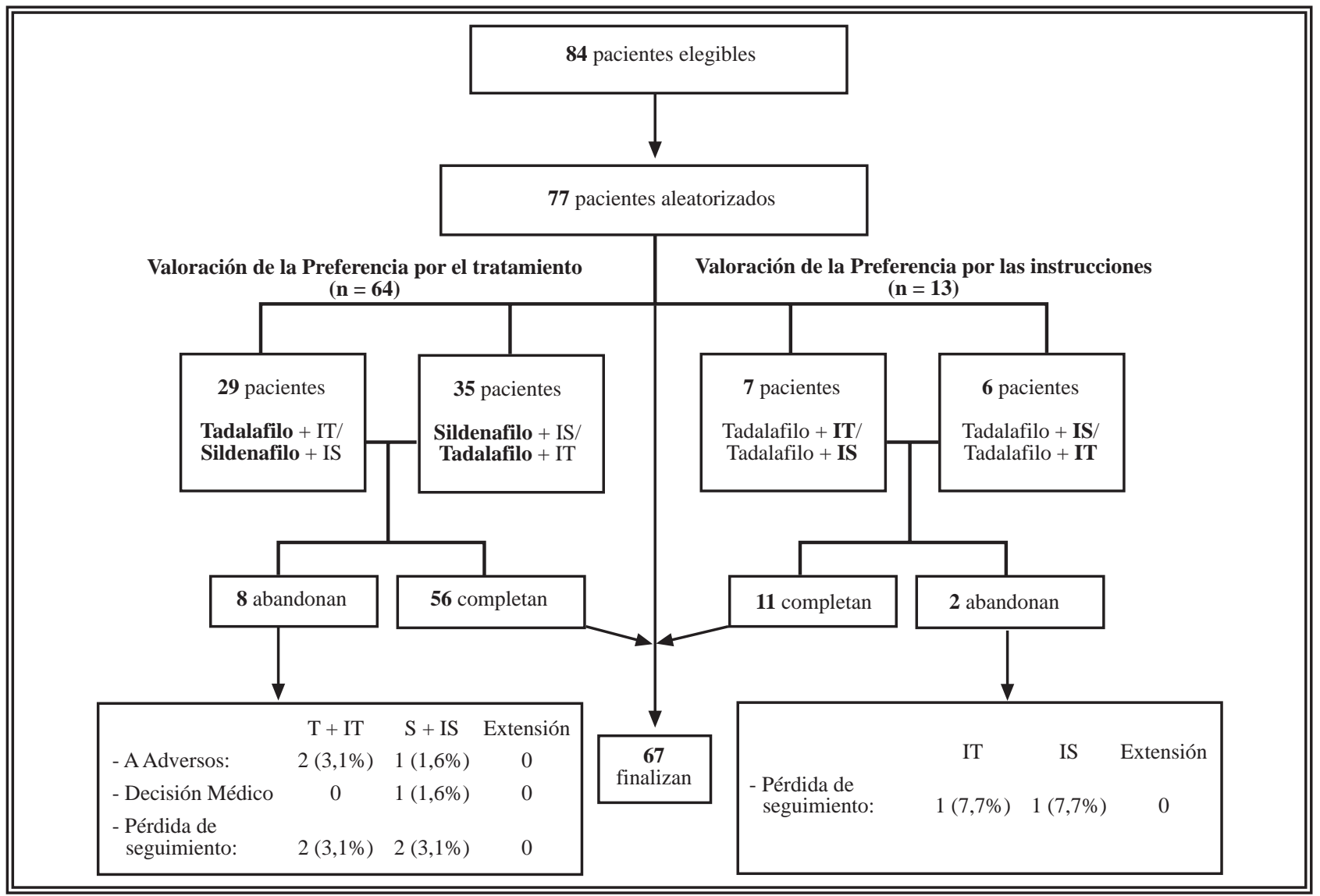

FIGURA 2. Disposición de los pacientes.

IT: Instrucciones de administración de Tadalafilo. IS: Instrucciones de administración de Sildenafilo. 
10 de los 77 pacientes elegibles abandonaron el estudio fueron: pérdida de seguimiento $(n=6)$, acontecimientos adversos $(n=3)$ y decisión del médico en el caso restante (Fig. 2). Ningún paciente abandonó por falta de eficacia.

La edad media de los pacientes participantes era de 52 años (rango de edad: 24-65). La mayoría (91\%) presentaban historial de disfunción eréctil de más de un año de duración, de etiología orgánica o mixta, fundamentalmente. Más de la mitad de los pacientes habían empleado sildenafilo con anterioridad (53\%). Se identificaron factores de riesgo cardiovascular comúnmente asociados a la DE, como hábito tabáquico (51,9\%), diabetes mellitus $(22,1 \%)$ o hipertensión arterial $(22,1 \%)$. Todas las características de los pacientes se describen en la Tabla 1.

Veintisiete $(45,8 \%)$ y siete $(58,3 \%)$ pacientes de las ramas de valoración PT y PIA respectivamente, solicitaron ajuste de dosis de entre los que acudieron a las visitas de titulación de dosis (59 en el grupo PT y 12 en PIA). Se concedió un ajuste de dosis al $81,5 \%$ de los 27 pacientes que lo solicitaron en el grupo de valoración de PT, si- guiendo las indicaciones de un máximo del 35\% por especificación del protocolo, de modo que, finalmente, de los 64 pacientes aleatorizados al grupo de valoración PT, 61 (95,3\%) recibieron sildenafilo 50 mg y $22(34,4 \%)$ recibieron sildenafilo $100 \mathrm{mg}$.

\section{PREFERENCIA}

\section{Variable principal}

Preferencia del paciente por la medicación

Un total de 56 de los 64 pacientes respondieron al final de la fase de tratamiento a la pregunta: "¿Qué régimen de tratamiento prefiere para la fase de extensión? (El recibido en el periodo 1/ periodo 2)". En base al porcentaje de respuestas, un número significativamente mayor de pacientes prefirieron recibir tadalafilo $(69,6 \%$ vs. $30,4 \%$ $(P<0,01)$ en la fase de extensión del estudio (Fig. 3).

Este patrón de preferencia mayoritaria por tadalafilo fue similar en todos los grupos del estudio, independientemente de la presencia de comorbilidad basal concomitante (hipertensión arterial, diabetes o enfermedad cardiovascular) el rango de edad del paciente o de la etiología o

Tabla 1

Características demográficas e historial clínico basal de los pacientes y causas de la disfunción eréctil (DE)

\begin{tabular}{|c|c|c|c|c|c|}
\hline & $\begin{array}{c}\text { T.+IT/ S.+IS } \\
(\mathrm{n}=29)\end{array}$ & $\begin{array}{c}\text { S.+IS / T.+IT } \\
(n=35)\end{array}$ & $\begin{array}{c}\mathrm{T}+\mathrm{IT} / \mathrm{T}+\mathrm{IS} \\
(\mathrm{n}=7)\end{array}$ & $\begin{array}{c}\mathrm{T}+\mathrm{IS} / \mathrm{T}+\mathrm{IT} \\
(\mathrm{n}=6)\end{array}$ & $\begin{array}{c}\text { Total } \\
(n=77)\end{array}$ \\
\hline \multicolumn{6}{|l|}{ Edad media, años } \\
\hline (max-min) & $\begin{array}{c}50,9 \\
(25,7-64,2)\end{array}$ & $\begin{array}{c}52,7 \\
(24,7-65,9)\end{array}$ & $\begin{array}{c}54,4 \\
(42,9-64,6)\end{array}$ & $\begin{array}{c}50,9 \\
(31,0-61,9)\end{array}$ & $\begin{array}{c}52,0 \\
(24,7-65,9)\end{array}$ \\
\hline \multicolumn{6}{|l|}{ Fumadores } \\
\hline No & $13(45 \%)$ & $15(43 \%)$ & $4(57 \%)$ & $4(67 \%)$ & $36(47 \%)$ \\
\hline Si & $16(55 \%)$ & 19 (54\%) & $3(43 \%)$ & $2(33 \%)$ & $40(52 \%)$ \\
\hline Desconocido & $0(0 \%)$ & $1(3 \%)$ & $0(0 \%)$ & $0(0 \%)$ & $1(1 \%)$ \\
\hline \multicolumn{6}{|l|}{ Etiologia DE, n (\%) } \\
\hline Psicógena & $1(3 \%)$ & $1(3 \%)$ & $1(14 \%)$ & $1(17 \%)$ & $4(5 \%)$ \\
\hline Orgánica & $20(69 \%)$ & 17 (49\%) & $3(43 \%)$ & $3(50 \%)$ & $43(56 \%)$ \\
\hline Mixta & $8(28 \%)$ & 17 (49\%) & $3(43 \%)$ & $2(33 \%)$ & $30(39 \%)$ \\
\hline \multicolumn{6}{|l|}{ Severidad DE, n (\%) } \\
\hline Leve & $5(17 \%)$ & $3(9 \%)$ & $1(14 \%)$ & $1(17 \%)$ & $10(13 \%)$ \\
\hline Moderada & $19(66 \%)$ & $21(60 \%)$ & $4(57 \%)$ & $3(50 \%)$ & 47 (61\%) \\
\hline Severa & $5(17 \%)$ & $11(31 \%)$ & $2(29 \%)$ & $2(33 \%)$ & $20(26 \%)$ \\
\hline \multicolumn{6}{|l|}{ Duración DE, n (\%) } \\
\hline$>=12$ Meses & $24(83 \%)$ & 33 (94\%) & $7(100 \%)$ & $6(100 \%)$ & 70 (91\%) \\
\hline Uso previo de sildenafilo, $n(\%)$ & $12(41 \%)$ & $23(65 \%)$ & $3(43 \%)$ & $3(50 \%)$ & $41(53 \%)$ \\
\hline \multicolumn{6}{|l|}{ Historial médico, n (\%) } \\
\hline Coronariopatías & $1(3 \%)$ & $2(6 \%)$ & $0(0 \%)$ & $0(0 \%)$ & $3(4 \%)$ \\
\hline Depresión & $1(3 \%)$ & $2(6 \%)$ & $0(0 \%)$ & $1(17 \%)$ & $4(5 \%)$ \\
\hline Diabetes mellitus & $4(14 \%)$ & $9(26 \%)$ & $2(29 \%)$ & $2(33 \%)$ & $17(22 \%)$ \\
\hline Hipertensión & $5(17 \%)$ & $8(23 \%)$ & $2(29 \%)$ & $2(33 \%)$ & $17(22 \%)$ \\
\hline
\end{tabular}

T: tadalafilo; S: Sildenafilo; IT: Instrucciones tadalafilo; IS: Instrucciones sildenafilo 


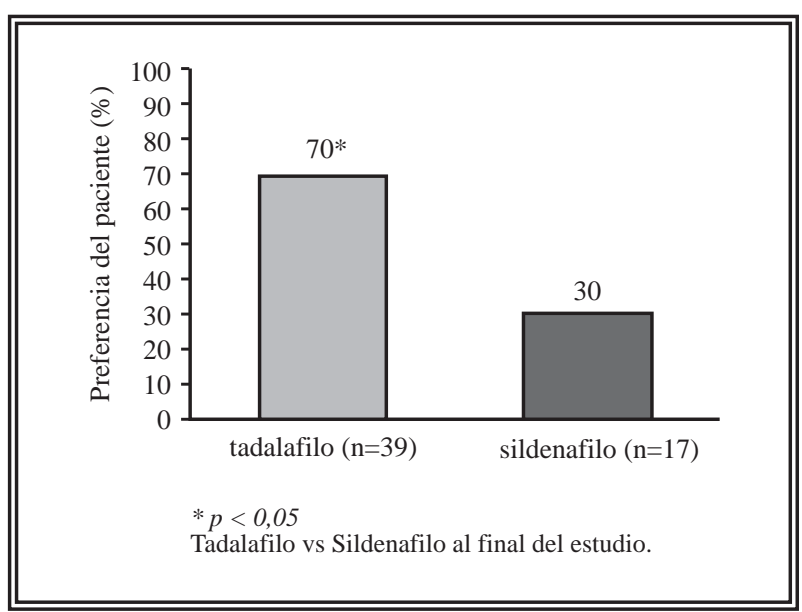

FIGURA 3. Preferencia de los pacientes por el tratamiento con tadalafilo + sus instrucciones frente al tratamiento con sildenafilo + sus instrucciones.

severidad de la DE al comienzo del estudio (Figs. 4A-4C). No obstante, tal y como se refleja en la Fig. 4A, la preferencia por tadalafilo fue significativamente más acusada en los pacientes $<50$ años (80,9\%), en aquellos con DE moderada $(79,4 \%)$ y de origen mixto $(75,0 \%)$. Igualmente, el uso previo o no de sildenafilo no ejerció ninguna influencia significativa sobre la elección del régimen preferido $(66,7 \%$ y $73,1 \%$ pacientes prefirieron tadalafilo, respectivamente), ni tampoco la

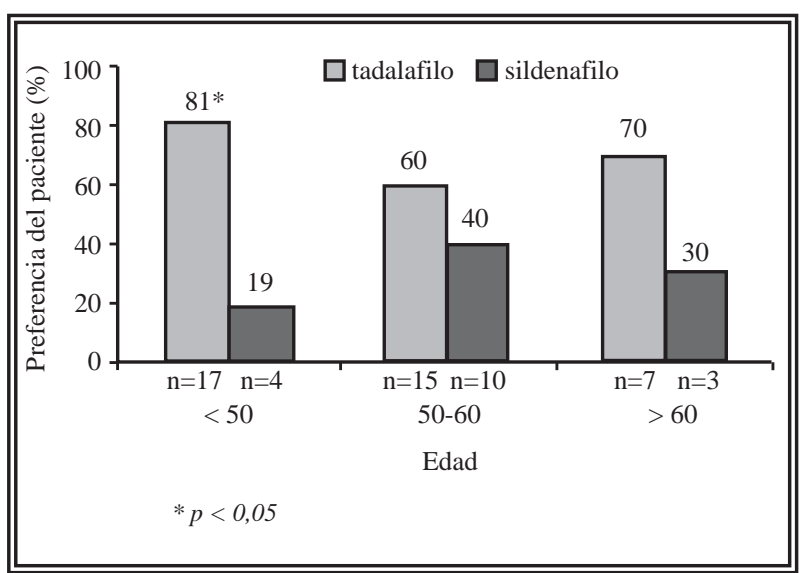

FIGURA 4A. Preferencia del paciente por el tratamiento con tadalafilo o con sildenafilo analizada por subgrupo de edad.

secuencia de tratamiento a la que los pacientes fueron aleatorizados (Figs. 4D y 4E). Por último, el requerimiento o no de ajuste de la dosis de sildenafilo (titulación), y el hecho de que éste les fuera concedido o no, no varió el resultado global de preferencia, de manera que una proporción aproximada de 3 de cada 4 pacientes prefirió tadalafilo frente a un 1 de cada 4 que prefirió sildenafilo. Sin embargo, 3 pacientes $(60,0 \%)$ a los que se denegó el aumento de dosis prefirieron sildenafilo frente a $2(40,0 \%)$ que prefirieron tadalafilo.

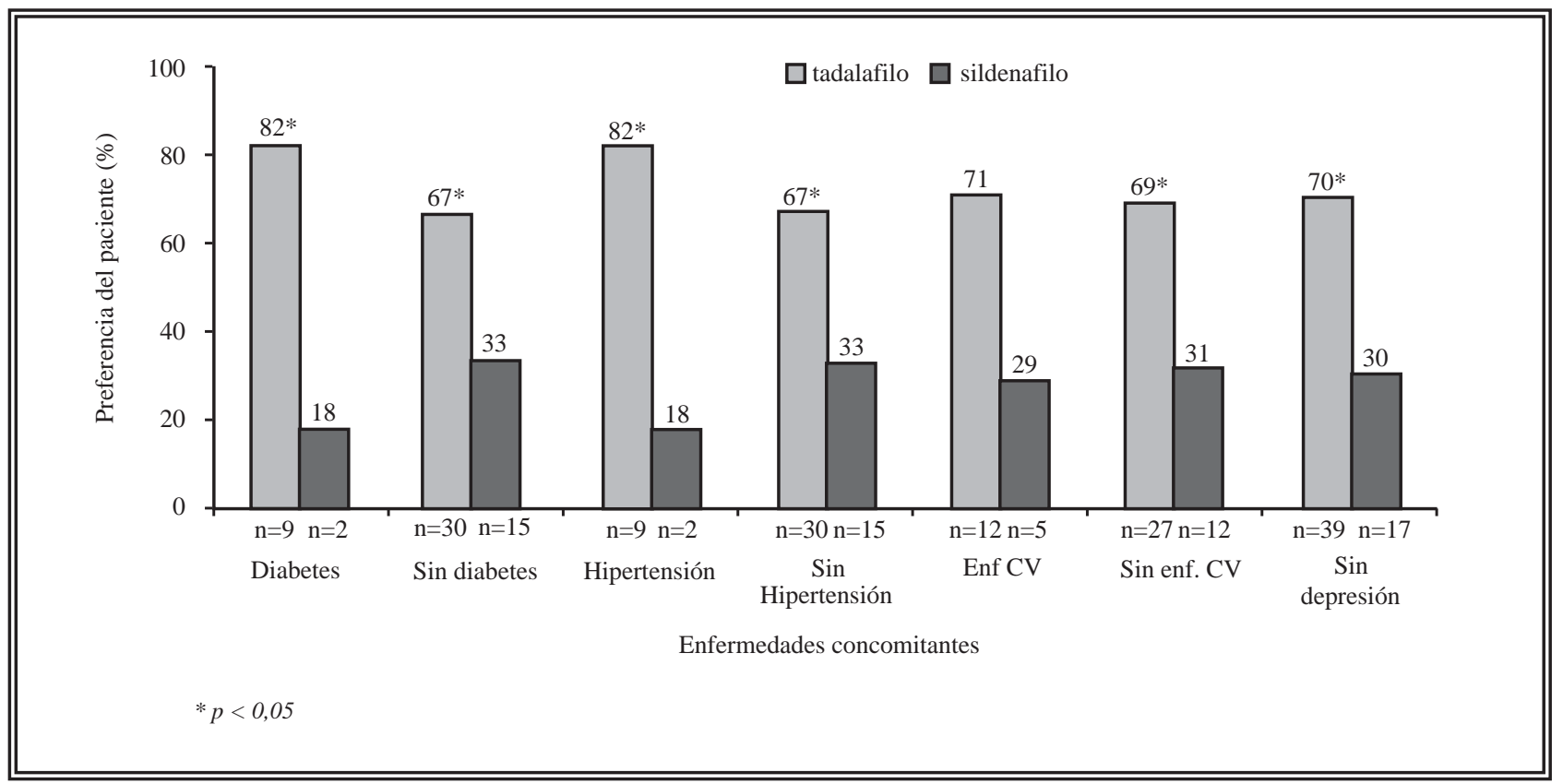

FIGURA 4B. Preferencia del paciente por el tratamiento con tadalafilo o sildenafilo analizada por subgrupo de enfermedades concomitantes. 


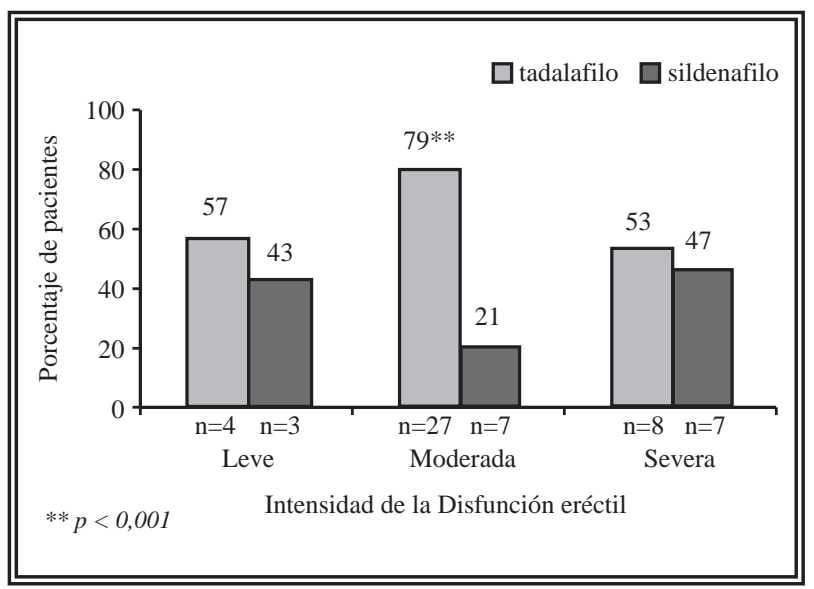

FIGURA 4C. Preferencia de los pacientes por el tratamiento con tadalafilo o con sildenafilo analizada según grado de la DE.

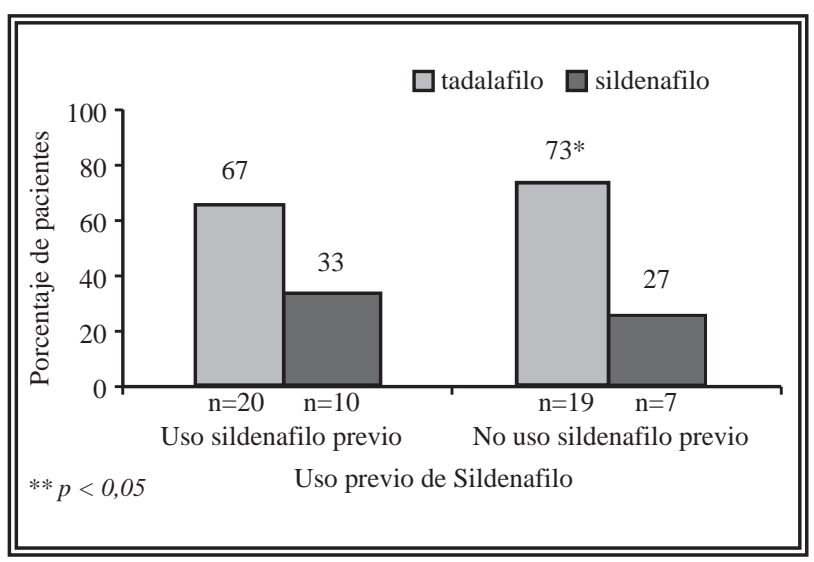

FIGURA 4D. Preferencia de los pacientes por el tratamiento con tadalafilo o con sildenafilo analizada según uso previo de sildenafilo.

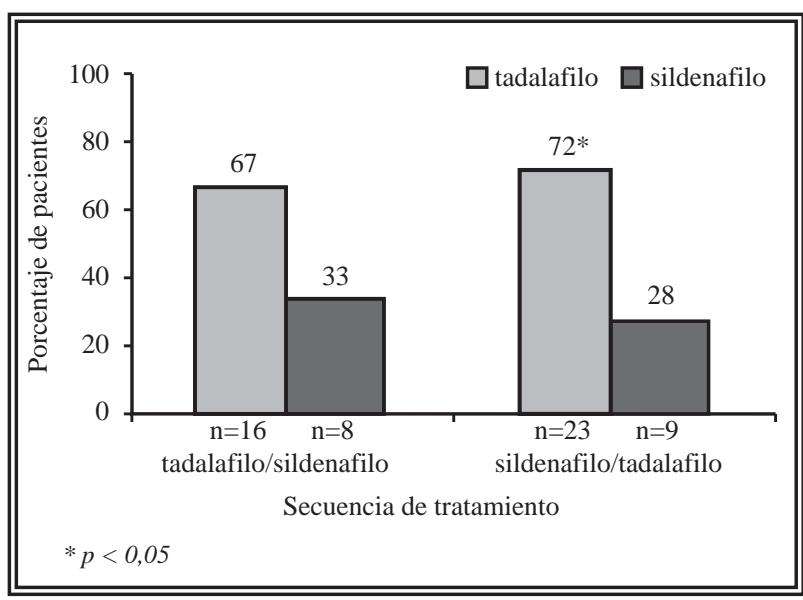

FIGURA 4E. Preferencia de los pacientes por el tratamiento con tadalafilo o con sildenafilo analizada según secuencia de tratamiento recibida.

\section{Variables secundarias}

Preferencia por las instrucciones de administración

Once de los 13 pacientes del grupo de valoración PIA respondieron a la pregunta sobre la opción elegida para la fase de extensión. De estos un porcentaje mayor de pacientes $(72,7 \% ; n=8)$ prefirió la opción correspondiente a las instrucciones de tadalafilo (Instrucciones T) frente a las de sildenafilo $(27,3 \%, \mathrm{n}=3$; Instrucciones $\mathrm{S})$ ( $>0,05)$. El orden en que habían recibido las secuencias de tratamiento (instrucciones $\mathrm{T} /$ instrucciones $\mathrm{S}$ o instrucciones $\mathrm{S} /$ instrucciones $\mathrm{T}$ ) no influyó en el resultado de preferencia por las instrucciones ( $p>0,05)$ (Fig. 5).

Número de tentativas de coito y su relación con la toma de la medicación

En los pacientes en los que se valoró la PT el número total de intentos de coito fue ligeramente superior mientras estaban recibiendo tadalafilo (1.531 intentos) que mientras recibían sildenafilo

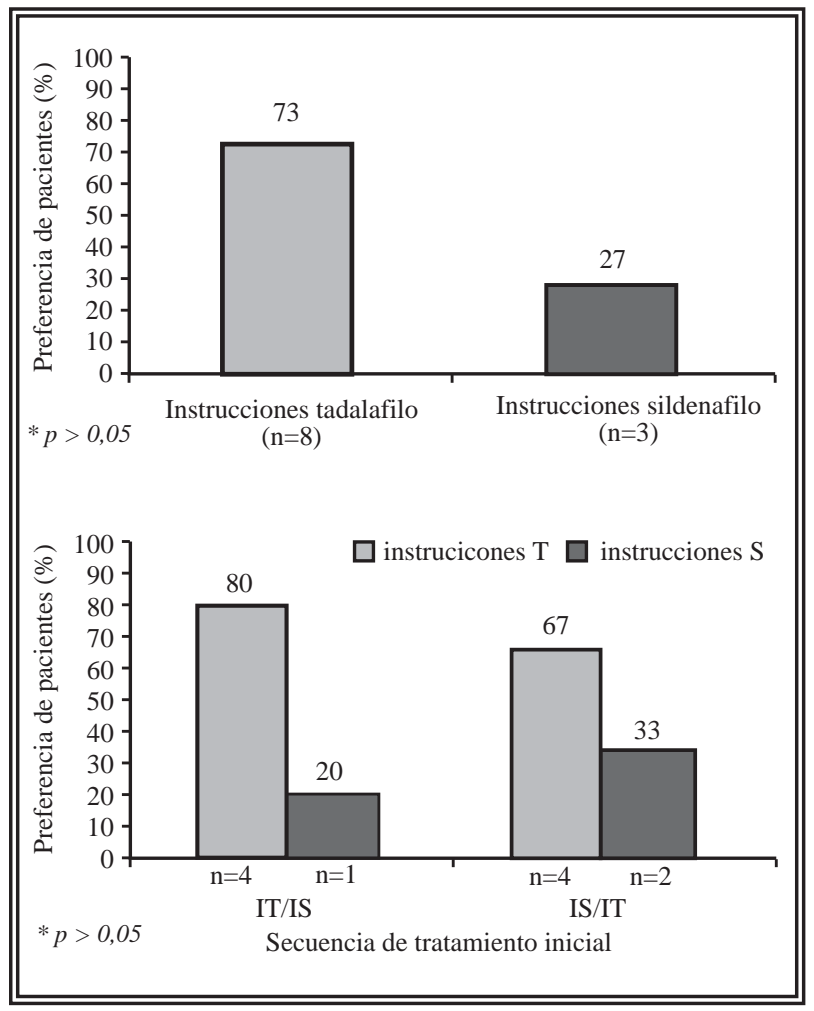

FIGURA 5. Preferencia de los pacientes por las instrucciones de sildenafilo o tadalafilo al final del estudio $y$ según el orden de secuencia de tratamiento recibido. 
(1.369 intentos). Un 51,6\% ( $\mathrm{n}=33)$ de los pacientes que recibieron tadalafilo en la Valoración de la Preferencia por la Medicación realizaron al menos un intento de coito transcurridas 12 horas o más tras la toma de tadalafilo, mientras que con sildenafilo lo hicieron un 23,4\% (n=15) de los pacientes. Considerando todos los intentos de coito con tadalafilo, el $58,3 \%$ de ellos tuvo lugar en las primeras 4 horas tras la toma, un $28 \%$ entre las 4 y las 12 horas y un 13,7\% después de 12 horas. Cuando los pacientes recibieron sildenafilo, el $85,1 \%$ de todos los intentos tuvieron lugar dentro de las primeras 4 horas, 9,5\% entre las 4 y 12 horas y un 5,4\% más allá de 12 horas tras la dosis. Por otra parte, en el grupo de valoración de las instrucciones también fue mayor el número de los intentos de relación sexual con las instrucciones de tadalafilo (341) que con sildenafilo (256), así como la proporción de pacientes que realizaron el intento de relación sexual transcurridas 12 h o más de la dosis $(46,2 \%$ con $\mathrm{T}$ $(n=6)$ vs $30,8 \%$ con $S(n=4)$.

\section{SEGURIDAD}

Tanto tadalafilo como sildenafilo fueron bien tolerados. En la valoración de la preferencia por la medicación, 3 pacientes $(4,7 \%)$ discontinuaron el tratamiento a causa de los acontecimientos adversos. Dos de éstos (insuficiencia cardiaca moderada y cefalea moderada) se comunicaron mientras estaban recibiendo el tratamiento con tadalafilo. El tercer paciente abandonó a causa de un infarto de miocardio agudo que sufrió mientras estaba tomando sildenafilo. De los anteriores, únicamente se consideró que la cefalea guardaba relación con el tratamiento en estudio. En la valoración de la preferencia por las instrucciones no se observó ningún abandono por culpa de los acontecimientos adversos.

En la valoración de la preferencia por el tratamiento los acontecimientos adversos más frecuentes fueron dispepsia, cefalea, síntomas gripales y dolor de espalda. En la Tabla 2 se muestran aquellos con una incidencia $\geq 2 \%$ aparecidos durante el tratamiento con tadalafilo y sildenafilo.

\section{Tabla 2}

Relación de acontecimientos adversos relacionados con el tratamiento comunicados con una incidencia $>2 \%$.

\begin{tabular}{lcccc} 
& \multicolumn{2}{c}{ T } & \multicolumn{2}{c}{ S } \\
& \multicolumn{2}{c}{$(\mathrm{n}=77)^{*}$} & \multicolumn{2}{c}{$(\mathrm{n}=64)$} \\
& $\mathrm{n}$ & $(\%)$ & $\mathrm{n}$ & $(\%)$ \\
\hline Dispepsia & 10 & 13,0 & 9 & 14,1 \\
Cefalea & 9 & 11,7 & 3 & 4,7 \\
Síntomas gripales & 5 & 6,5 & 6 & 9,4 \\
Dolor de espalda & 4 & 5,2 & 1 & 1,6 \\
Mialgia & 3 & 3,9 & & \\
Dolor abdominal & & & & \\
superior & 3 & 3,9 & & \\
Diarrea & & & & \\
Nauseas & & & & \\
Rinitis & 2 & 2,6 & & \\
Molestias estomacales & 1 & 1,3 & & \\
\hline
\end{tabular}

T: tadalafilo*(incluyendo los 13 pacientes de PIA); S: sildenafilo

En la valoración de la preferencia por las instrucciones de administración, los acontecimientos adversos más frecuentes fueron síntomas gripales (15\%), dispepsia $(7,7 \%)$, y cefalea $(7,7 \%)$, aunque debido al pequeño tamaño muestral $(n=13)$ se debe cuestionar su representatividad.

En general, la tolerabilidad de ambos medicamentos fue buena y similar a la observada en la población del estudio global (EE.UU. y Alemania), salvo el síndrome gripal que se comunicó con más frecuencia en la muestra española.

\section{DISCUSIÓN}

Este estudio recoge los resultados obtenidos de una cohorte de 77 pacientes reclutados en España, participantes en un estudio multicéntrico, doble ciego, aleatorizado, cruzado que incluyó un total de 265 pacientes en EE.UU. y Europa. Las características basales de la población española son comparables a la de la muestra global, salvo por un porcentaje de fumadores superior en 
la muestra española (52\% frente al 29\% en la muestra global) que es característico de la epidemiología de la DE en España ${ }^{5}$.

En la variable primaria del estudio, la preferencia por la medicación, el 69,6\% de los pacientes prefirieron tadalafilo frente a un $30,4 \%$ que prefirieron recibir sildenafilo en la fase de extensión $(\mathrm{P}<0,01)$. Estos porcentajes son similares a los observados en la población global del estudio (73\% y 27\% respectivamente, para tadalafilo y sildenafilo $\mathrm{P}<0,01)$. El test chi-cuadrado no mostró diferencias estadísticas en la distribución de la proporción de preferencia entre pacientes españoles y no españoles $(\mathrm{P}>0,05)$.

Asimismo, en la evaluación de Preferencia por las Instrucciones de Administración en la cohorte de 13 pacientes que recibieron tadalafilo con las instrucciones propias o con las de sildenafilo, la mayoría de los pacientes españoles $(72,7 \%)$ prefirió las instrucciones de administración propias de tadalafilo, si bien el reducido tamaño muestral de esta cohorte no permite alcanzar niveles de significación estadística $(p>0,05) \mathrm{El}$ porcentaje de preferencia por el tratamiento con tadalafilo analizado por subgrupos no varió en función de las enfermedades concomitantes basales, el uso previo de sildenafilo, o la secuencia de tratamiento o ajuste de dosis recibidas. Tampoco en función de la edad o el origen y grado de severidad basal de la DE, aunque en la muestra española se observó una diferencia más acusada en el subgrupo de pacientes con edad $<50$ años, en aquellos con DE de intensidad moderada, o con DE de origen orgánico o mixto. Es importante destacar que la preferencia por tadalafilo se mantuvo tanto en los pacientes con exposición previa a sildenafilo antes del estudio $(66,7 \%)$ como en los que no lo habían utilizado nunca $(73,1 \%)$. Por otra parte, con tadalafilo el número de coitos fue más numeroso y transcurrió más tiempo desde la toma de la medicación y la primera tentativa de coito que con sildenafilo, de modo que aproximadamente la mitad de las tentativas de coito de los pacientes que recibieron tadalafilo tuvo lugar más allá de las primeras 4 horas tras la toma. Este comportamiento sin duda responde al hecho de que el singular perío- do de respuesta de tadalafilo permite a los pacientes iniciar la actividad sexual en diferentes momentos tras la toma a lo largo de 36 horas, y no necesariamente en las primeras 4 horas como es lo esperable con fármacos de acción más corta.

No obstante, a pesar de unas instrucciones de administración más restrictivas en cuanto al período de respuesta se refiere, casi la cuarta parte de los sujetos que recibieron sildenafilo realizó al menos un coito transcurridas 12 ó más horas tras la dosificación, evidenciándose con ello el potencial efecto beneficioso que perciben muchos pacientes en el hecho de poder distanciar lo máximo posible la actividad sexual con respecto a la toma del fármaco.

En general, la tolerabilidad de ambos medicamentos fue buena y similar entre ambos fármacos y consistente con la observada en la población del estudio global, (salvo el sindrome gripal que se comunicó con más frecuencia en la muestra española), y con el perfil general de eventos adversos comunicados con tadalafilo y sildenafi$10^{8,9,11-14}$. Únicamente tres pacientes $(4,7 \%)$ abandonaron por acontecimientos adversos (2 con tadalafilo y 1 con sildenafilo).

Si bien los resultados de este estudio son claros y consistentes con los observados en la mayor parte de los estudios de preferencia entre ambos fármacos publicados hasta la fecha ${ }^{20-24}$, es necesario tener en cuenta una serie de factores a la hora de interpretar los datos del estudio:

$\mathrm{El}$ acotamiento del 35\% de pacientes a los que se permitiría recibir sildenafilo $100 \mathrm{mg}$ podría ejercer algún tipo de influencia en el resultado final del estudio pues, aun cuando la literatura médica respalda este supuesto ${ }^{25-27}$ no existe certeza absoluta de que refleje exactamente la proporción de pacientes que emplean sildenafilo 50 ó 100 mg en la práctica clínica. Además un porcentaje no despreciable de pacientes que solicitaron el cambio de dosis no recibieron el tratamiento deseado porque se había alcanzado el limite. No obstante, el resultado global en cuanto a preferencia a favor de tadalafilo está respaldado por el hecho de que hubo una preferencia significativa por tadalafilo en el grupo de pacientes que no solicitaron el ajuste de dosis y porque la 
totalidad de los pacientes que solicitaron el ajuste de dosis y se les concedió prefirieron también tadalafilo. Sólo en los 5 pacientes españoles cuya solicitud de aumento de dosis no fue atendida se decantó la preferencia a favor de sildenafilo, aunque la diferencia no tuvo significado estadístico dado el pequeño tamaño del subgrupo analizado.

Es preciso mencionar también que en el estudio se ha empleado únicamente la dosis tadalafilo $20 \mathrm{mg}$ ya que era la que, en el momento en que se desarrolló este estudio, se consideraba como dosis de inicio recomendada. En la actualidad, si bien la dosis de inicio recomendada en algunos países es, efectivamente la de $20 \mathrm{mg}$, en Europa es la de $10 \mathrm{mg}$. El hecho de incluir a pacientes con experiencia de uso previo con sildenafilo pudo influir en la preferencia por el tratamiento con tadalafilo, aunque de éstos se desconoce cuantos no habian respondido previamente a sildenafilo de los que sí lo habían hecho.

Asimismo, las instrucciones de dosificación empleadas pueden introducir algún sesgo a favor de tadalafilo en la interpretación de la información dada al paciente, pues al ser una propuesta de instrucciones (ya que el fármaco aún no estaba comercializado) introducían ciertos términos que sugerían la flexibilidad de la toma y la posibilidad de espontaneidad en la relación sexual mientras que las instrucciones de sildenafilo fueron las de fabricante ya autorizadas por las autoridades regulatorias. Por otra parte, a los pacientes tratados con sildenafilo se les entregó solamente las instrucciones de administración de sildenafilo y no las de tadalafilo, si bien se hizo para evitar un sesgo de eficacia contrario a sildenafilo que hubiera supuesto fomentar su empleo fuera de la ventana temporal de máxima eficacia.

A pesar de todas las consideraciones anteriores, estos resultados demuestran que en condiciones de doble ciego los pacientes prefirieron mayoritariamente el tratamiento con tadalafilo para la disfunción eréctil. En el estudio, no se evaluaron específicamente las razones de esta preferencia, si bien sí se ha realizado en otros estudios publicados ${ }^{25-26}$ siendo el mayor período de respuesta la razón principal esgrimida para justificar la elección.
En conclusión, el 69.6\% de los pacientes españoles que participaron en la valoración de preferencia por un tratamiento prefirieron tadalafilo a sildenafilo para el tratamiento de su disfunción eréctil. De igual modo, el 72,7\% de los pacientes en que se evaluó la preferencia por las instrucciones de administración eligió tomar tadalafilo con las instrucciones de tadalafilo, en vez de con las de sildenafilo.

Potenciales limitaciones del estudio incluyen la restricción de acceso del $35 \%$ a la dosis de 100 mg de sildenafilo y unas instrucciones de tadalafilo más detalladas, si bien ambos son de dudoso impacto en los resultados. Tanto tadalafilo como sildenafilo fueron, en general, bien tolerados.

Los datos refuerzan la necesidad de involucrar, en la medida de lo posible, al paciente y su pareja en la toma de decisiones en el abordaje de la $\mathrm{DE}$, e individualizar el tratamiento tras implementar una estrategia adecuada de información sobre los beneficios y características de los distintos tratamientos y de identificación de los factores sociológicos, psicológicos, culturales y relacionados con los hábitos sexuales de las parejas. De este modo se garantizará que la opción final de tratamiento sea realmente la que más se ajuste a las necesidades y expectativas del paciente y su pareja.

Agradecimientos. Este estudio clínico ha sido realizado con el patrocinio de Lilly ICOS LLC. Los autores desean agradecer al Prof. Remigio Vela Navarrete, Dr. José M. Martínez-Sagarra, Dr. Alberto Gonzalvo, Dr. Juan Vicente Cardoso, Dr. Carlos Llorente, y Dr. Javier Estébanez, su participación en el estudio y aportación de datos científicos para la realización del mismo, y a Beatriz Sanz, María Costi, y Elena Bolaños, por su trabajo, dedicación y ayuda para llevar a cabo el estudio.

\section{REFERENCIAS}

1. NIH Consensus Development panel on Impotence. Impotence. JAMA 1993;270:83-90.

2. Leiblum SR. After sildenafil: bridging the gap between pharmacologic treatment and satisfying sexual relationships. J Clin Psychiatry. 2002 Dec;63(12):17-22.

3. Pommerville P. Erectile dysfunction: An overview. Can J Urol 2003;10(suppl.1):2-6.

4. World Population Projection Program of United Nations (2002 Revision). 
5. Martín-Morales A, Sánchez-Cruz JJ, Saenz de Tejada I, Rodríguez-Vela L, Jiménez-Cruz JF, Burgos-Rodríguez R. Prevalence and independent risk factors for erectile dysfunction in Spain: results of the Epidemiologia de la Disfuncion Erectil Masculina Study. J Urol 2001 Aug;166(2):569-574; discussion 574-575.

6. Aytac IA, McKinlay JB, Krane RJ. The likely worldwide increase in erectile dysfunction between 1995 and 2025 and some possible policy consequences. Br J Urol Int 1999;84:450- 456.

7. Wespes E, Amar E, Hatzichristou D, Montorsi F, Pryor J, Vardi Y. European Asociation of Urology. Guidelines on erectyle dysfunction. March 2004.

8. Goldstein I, Lue TF, Padma-Nathan H, Rosen RC, Steers WD, Wicker PA. Oral sildenafil in the treatment of erectile dysfunction. N Engl J Med 1998;338:1397-1404.

9. Sildenafil Citrate (Viagra1) US Package Insert. Nueva York: Pfizer, Inc 2002.

10. Seftel AD. Challenges in oral therapy for erectile dysfunction. J Androl 2002;23:729-736.

11. Ficha Técnica de Tadalafilo, 2004

12. Brock GB, McMahon CG, Chen KK, Costigan T, Shen W; Watkins $\mathrm{V}$ et al. Efficacy and safety of tadalafil for the treatment of erectile dysfunction: results of integrated analyses. J Urol 2002;168(4 Pt 1):1332-1336.

13. Carson CC, Rajfer J, Eardley I, Carrier S, Denne JS, Walker DJ, et al. The efficacy and safety of tadalafil: an update. BJU International 2004;93:1276-1281.

14. Montorsi F, Verheyden B, Jünemann KP, Moncada I, Valiquette L, Denne J. Long-Term Safety and Tolerability of Tadalafil in the Treatment of Erectile Dysfunction. European Urology 2004;45:339-345.

15. Porst H, Padma-Nathan H, Giuliano F, Anglin G, Varanese L, Rosen R. Efficacy of tadalafil for the treatment of erectile dysfunction at 24 and 36 hours after dosing: a randomized controlled trial. Urology. 2003 Jul;62(1):121-125; discussion 125-126.

16. Raymond C. Rosen PhD, Harin Padma-Nathan, MD, Ridwan Shabsigh, MD, Khalil Saikali, PhD, Exec MB, Vish Watkins, MD et al. Determining the Earliest Time within 30 Minutes to Erectogenic Effect after Tadalafil 10 and 20 mg: A Multicenter, Randomized,Double-Blind, PlaceboControlled, At-Home Study. J Sex Med 2004;1:193-200.

17. Young Y, Feldman RF, Kaufman JK, Averbach SA, Ahuja SA, Purphy AM, et al. Tadalafil 10 and $20 \mathrm{mg}$ Effectively Treat Erectile Dysfunction for up to 36 Hours. J Sex Med 2004;1(suppl1):48,Abstract O61.
18. Chen J, Habjeesh NJ, Greenstein A. Sildenafil versus the vacuum erection device. Patient preference. J urol 2001;166: 1779-1781.

19. Hatzichristou DG, Apostolidis A, Tzortzis V, Ioannides E, Yannakoyorgos K, Kalinderis A. Sildenafil versus intracavernous injection therapy: efficacy and preference in patients on intracaver-nous injection for more than 1 year. J Urol 2000; 164:1197-1200.

20. Stroberg P, Murphy A, Costigan T. Switching patients with erectile dysfunction from sildenafile citrate to tadalafil: Results of a European multicenter, open-label study of patient preference. Clinical Therapeutics 2003;25(11): 2724-2737.

21. Govier F, Potempta AJ, Kaufman J, Denne J, Kovalenko P, Ahuja S. A multicenter, randomized, double-blind, crossover study of patient preference for Tadalafil $20 \mathrm{mg}$ or Sildenafil citrate $50 \mathrm{mg}$ during initiation of treatment for erectile dysfunction. Clinical Therapeutics 2003;25(11): 2709-2723.

22. Porst H, Kleingarn M, Arnds S. The two PDE 5 inhibitors sildenafil and tadalafil: results of an independent intraindividual comparative trial [abstract]. Eur Urol Suppl 2004; $3(2): 27$.

23. Porst H, Arnds S, Kleingarn M. The 3 PDE 5 inhibitors sildenafil, tadalafil and vardenafil: results of an independent intraindividual comparative study [abstract]. Eur Urol Suppl 2004;3(2):104.

24. Von Keith A, Rajfer J, Segal S, Murphy A, Denne J, Costigan $\mathrm{T}$, et al. A multicenter, randomized, double-blind, crossover study to evaluate patient preference between Tadalafil and Sildenafil. European Urology 2004;45:499-509.

25. El Galley R, Rutland H, Talic R, Keane T, Clark H. Longterm efficacy of sildenafil and tachyphylaxis effect. J Urol 2001;166:927-931.

26. Marks LS, Duda C, Dorey FJ, Macairan ML, Santos PB. Treatment of erectile dysfunction with sildenafil. Urology 1999;53:19-24

27. Orrico KB, Verdiano RM, Wohl LB. Sildenafil Dose Titration Program. Am J Health Syst Pharm 1998;55:2297.

Dr. A. Cassinello Hervas

Laboratorios Lilly, S.A.

Avda. de la Industria, 30

28108 Alcobendas (Madrid)

(Trabajo recibido el 12 de julio 2005) 